\title{
Implementing Unitarity in Perturbation Theory
}

\author{
C.S. Lam \\ Department of Physics, McGill University \\ 3600 University St., Montreal, Q.C., Canada H3A 2T8 \\ Email:LLam@physics.mcgill.ca
}

(November 6, 2018)

\begin{abstract}
Unitarity cannot be perserved order by order in ordinary perturbation theory because the constraint $U U^{\dagger}=\mathbf{1}$ is nonlinear. However, the corresponding constraint for $K=\ln U$, being $K=-K^{\dagger}$, is linear so it can be maintained in every order in a perturbative expansion of $K$. The perturbative expansion of $K$ may be considered as a non-abelian generalization of the linked-cluster expansion in probability theory and in statistical mechanics, and possesses similar advantages resulting from separating the short-range correlations from longrange effects. This point is illustrated in two QCD examples, in which delicate cancellations encountered in summing Feynman diagrams of are avoided when they are calculated via the perturbative expansion of $K$. Applications to other problems are briefly discussed.
\end{abstract}

Probability conservation is not maintained order by order in ordinary perturbation theory. This happens because the unitarity relation $U U^{\dagger}=\mathbf{1}$ (for the time-evolution operator $U)$ is nonlinear, whereas the constraints for the other exact conservation laws, such as energy, momentum, and charge, are linear. Order-by-order probability conservation can be restored if we expand instead $K=\ln U \equiv \sum_{n \geq 1} K_{n} / n$, for then the unitarity constraint becomes the linear constraint $K=-K^{\dagger}$. As long as every $K_{n}$ is kept to be anti-hermitean, $U=\exp (K)$ will be unitary no matter where the $K$-expansion is truncated. We shall henceforth refer to 
the perturbation theory in which $K=\ln U$ is expanded as the unitary perturbation theory. Mathematically, such an expansion is known as the Magnus expansion [1].

Besides unitarity, there is another reason to deal with the perturbative expansion $K_{n}$ of $K=\ln U$, rather than $U=1+\sum_{n \geq 1} U_{n}$, because the former is the generalization of the linked-cluster expansion in probability theory and in statistical mechanics [2], with the associated advantages in separating short-range correlations from the long-range effects. It is also analogous to the expansion of the effective potential in quantum field theory. The added complication here is that we are dealing with non-commutative operators rather than c-number functions. In QCD, short-range correlations refer to correlations in time and in color. This point will be explained later with two QCD examples.

Unitary perturbation theory can also be regarded as an extension of the familiar eikonal approximation. For elastic scattering of a high-energy particle from a static potential, the scattering amplitude is diagonal in energy and the impact parameter. The scattering amplitude $\langle U\rangle$ to all orders can be summed up to the form $\left\langle\exp \left(K_{1}\right)\right\rangle=\exp \left(\left\langle K_{1}\right\rangle\right)$, where $\left\langle K_{1}\right\rangle$ is equal to the first Born approximation $\left\langle U_{1}\right\rangle$, and $\langle\cdots\rangle$ is the matrix element in impact-parameter space [3]. This eikonal approximation is also valid for electron-electron scattering when fermion loops are neglected [5]. For high-energy scattering of a quark from a color potential, amplitudes of all orders can again be summed up into the form $\langle\exp (K)\rangle=\exp (\langle K\rangle)$, but now $\langle K\rangle$ is a color matrix, with $\left\langle K_{n}\right\rangle$ proportional to the $n$th nested commutator of the color matrices $t_{a}$ [6]. In particular, $\left\langle K_{n}\right\rangle \neq 0$ even for $n>1$. Therefore unitary perturbation theory can be thought of as a generalization of the nonabelian eikonal approximation.

The relation between $U_{n}$ and $K_{n}$ can be obtained by expanding the exponential $\exp \left(\sum K_{n} / n\right)$ to the $n$th order. One obtains

$$
U_{n}=\sum_{(m)} \frac{1}{k !} \frac{1}{\prod_{i=1}^{k} m_{i}} K_{m_{1}} K_{m_{2}} \cdots K_{m_{k}}, \quad \text { unk }
$$

where the sum is taken over all partition $(m)=\left(m_{1} m_{2} \cdots m_{k}\right)$ of the integer $n$. If $U$ is the time-evolution operator between times $T^{\prime}$ and $T$, ordinary perturbation theory tells us 


$$
U_{n}=(-i)^{n} \int_{\mathcal{R}_{n}} d^{n} t H_{I}\left(t_{1}\right) \cdots H_{I}\left(t_{n}\right), \quad \text { uh }
$$

where $\mathcal{R}_{n}$ is the hyper-triangular integration region $\left\{T \geq t_{1} \geq \cdots t_{n} \geq T^{\prime}\right\}$. Together with (1), this may be used to derive a dynamical expression for $K_{n}$. In particular,

$$
\begin{aligned}
& K_{1}=U_{1}=-i \int_{T^{\prime}}^{T} d t H_{I}(t) \\
& K_{2}=2 U_{2}-U_{1}^{2}=(-i)^{2} \int_{T^{\prime}}^{T} d t_{1} \int_{T^{\prime}}^{t_{1}} d t_{2}\left[H_{I}\left(t_{1}\right), H_{I}\left(t_{2}\right)\right] . \quad k 12
\end{aligned}
$$

The difference between ordinary and unitary perturbation theories can be illustrated by a simple spin- $\frac{1}{2}$ example [7], with interaction $-\frac{1}{2} \vec{\sigma} \cdot \vec{B}(t)$, and an external magnetic field $\vec{B}(t)=\left(B_{\perp} \cos \omega t,-B_{\perp} \sin \omega t, B_{0}\right)$. The longitudinal field $B_{0}$ causes a Zeeman splitting of magnitude $B_{0}=\hbar \omega_{0}$; the transverse field $B_{\perp}$ induces a transition between the lower and the upper states. Assuming the system to occupy the lower state initially, the probability to be in the upper state is plotted in Fig. 1, as a function of the scaled frequency variable $\Omega=\hbar\left(\omega-\omega_{0}\right) / B_{\perp}$ at the scaled time $\tau=B_{\perp} t / \hbar=\pi$. The solid, deahed, dash-dot, and dotted curves are respectively the exact solution, the first Born approximation $P_{1}=\left\langle U_{1}\right\rangle$, the first unitary approximation $U P_{1}=\left\langle\exp \left(K_{1}\right)\right\rangle$, and the second unitary approximation $U P_{2}=$ $\left\langle\exp \left(K_{1}+K_{2} / 2\right)\right\rangle$. The notation $\langle\cdots\rangle$ represents the matrix element between the upper and the lower states. We see from Fig. 1 that the probability given by the Born approximation is larger than 1 for small $|\Omega|$, violating unitarity, but both unitary approximations stay within the unitarity bound. As a function of $\tau$ at the resonance frequency $\Omega=0$, the exact solution as well as the two unitary approximations are periodic, with a period $2 \pi$ oscillating between 0 and 1, whereas the Born approximation grows monotonically like $\tau^{2}$, making it a worse and worse approximation at large $\tau$.

For QCD, the unitary perturbation theory can be used to classify unitary parton-parton elastic amplitudes and to produce unitary model for Pomeron amplitudes [11].

Besides unitarity, it is often more profitable to compute $U_{n}$ via $K_{n}$, because $K_{n}$ contains short-time and color correlations via the appearance of the commutator of the $H_{I}$ 's, instead of their product in $U_{n}$. I will illustrate this remark with two second-order QCD examples. 
Higher-order situtations will be discussed later.

The first example concerns high-energy electron-electron and quark-quark elastic scattering near the forward direction, with Mandelstam variables $s$ and $t$. The only difference between the two cases is the presence of a color matrix $t_{a}$ at the vertices of QCD diagrams, Fig. 2(a),(b),(c). The formulas for quark-quark amplitudes [8] are equally applicable to electron-electron amplitudes if we replace $t_{a}$ by 1 . All amplitudes have a common factor $i s / 2 m^{2}$ which will be factored out, leaving the rest to be $\langle U\rangle$. Fig. 2(a) gives the first Born amplitude $\left\langle U_{1}\right\rangle=\left(i g^{2} / \Delta^{2}\right) G_{1}$, where $\Delta$ is the (transverse) momentum transfer with $t=-\Delta^{2}$, and $G_{1}=t_{a} \times t_{a}$ is the color factor of Fig. 2a. The amplitude for Fig. 2(b) is $\left(-i g^{4} / 2 \pi\right) I_{2} \ln \left(s e^{-\pi i}\right) G_{2}$, and that for Fig. $2(\mathrm{c})$ is $\left(i g^{4} / 2 \pi\right) I_{2} \ln s\left(G_{2}+G_{1} N_{c} / 2\right)$, where $I_{2}=\int d^{2} k_{\perp} /\left[(2 \pi)^{2} k_{\perp}^{2}\left(\Delta-k_{\perp}\right)^{2}\right]$. The color factor $G_{2}=G_{1} \times G_{1}$ is that of Fig. 2(b), with $N_{c}$ being the number of colors in the theory. This number is to be set equal to 0 for QED. The second Born amplitude, being the sum of Figs. 2(b) and 2(c), is $\left\langle U_{2}\right\rangle=\left(-i g^{4} / 2 \pi\right) I_{2}\left(-\pi i G_{2}-\ln s G_{1} N_{c} / 2\right)$. Note the cancelation of the $\ln s$ factor in the term proportional to $G_{2}$. The $G_{1}$ term consists of a color-octet exchange. It is not negligible compared to the first Born amplitude $\left\langle U_{1}\right\rangle$ in the leading-log approximation where $g^{2} \ll 1$ and $g^{2} \ln s=O(1)$. In fact it is the beginning of the reggeized gluon contribution. The color factor $G_{2}=G_{1} \times G_{1}$ consists of two color-octet exchanges, which can be decomposed into a color-octet and a color-singlet. Its coefficient in the impact-parameter representation is equal to the coefficient of $G_{1} \times G_{1}$ in $\frac{1}{2}\left\langle U_{1}^{2}\right\rangle$, a factor of $\ln s$ down from the coefficient of the $G_{1}$ term. Its color-octet contribution is therefore negligible in the leading-log approximation, but its color-singlet component is leading and constitute the beginning of the Pomeron amplitude. For QED, the factor $N_{c}$ in front of $G_{1}$ is zero, and $G_{2}$ is replaced by 1 . Hence $\left\langle U_{2}\right\rangle=\frac{1}{2}\left\langle U_{1}\right\rangle^{2}$ in the impact-parameter representation.

To summarize, a delicate cancelation of ln s occurs between Figs. 2(b) and 2(c) in QED, and in the color-singlet channel of QCD. This is reminiscent of the delicate cancellation of the volume factor in the grand partition function in statistical mechanics unless the linkedcluster expansion is used [2]. As a result, the $k$ color-octet exchange amplitude in QCD 
is proportional to $g^{2 k}\left(g^{2} \ln s\right)^{2-k}(k=1,2)$, and it is this dependence that leads to the reggeon amplitudes in QCD. We shall now see that if we calculate $\left\langle U_{2}\right\rangle$ via $\left\langle K_{1}^{2}\right\rangle$ and $\left\langle K_{2}\right\rangle$, then delicate cancelation is not needed, and the energy dependence needed for the reggeon structure becomes immediate.

Since $\left\langle K_{1}\right\rangle=\left\langle U_{1}\right\rangle$, it is given simply by Fig. 2(a). For $\left\langle K_{2} / 2\right\rangle$, it can be computed from Fig. 2(c), provided the product of the color matrices $t_{a} t_{b}$ in the upper quark line is replaced by their commutator $\left[t_{a}, t_{b}\right]\left[6\right.$, thus making the color factor purely $G_{1}$. Otherwise, the result is identical to that of Fig. 2(c). In the impact-parameter representation where $K_{i}$ are diagonal, we obtain in this way the same result as before: $\left\langle K_{2} / 2\right\rangle$ is given by the $G_{1}$ coefficient of Fig. 2(c), or that of $\left\langle U_{2}\right\rangle$, and $\left\langle K_{1}\right\rangle^{2} / 2$ is given by the $G_{2}=G_{1} \times G_{1}$ coefficient of $\left\langle U_{2}\right\rangle$. The absence of the $\ln s$ factor in $\left\langle K_{1}\right\rangle^{2} / 2$ is not due to cancelation; it is simply a consequence that $\left\langle K_{1}\right\rangle$ is independent of $s$. This is the same as in statistical mechanics when linked cluster expansion is used [2]. The reggeized gluon contribution is now isolated in $\left\langle K_{2} / 2\right\rangle$ alone, without having to combine two Feynman diagrams to obtain it. Its octet origin can be traced back to the color commutator in the upper quark line, or the commutator structure of $K_{2}$ shown in (3). This gives $\left\langle K_{2}\right\rangle$ a physical meaning as (part of) the one-reggeon amplitude.

The second example is pion-nucleon elastic scattering in the large- $N_{c}$ limit. The tree diagrams are shown in Fig. (d),(e), with pion-quark interaction given by a Hamiltonian of the form $H_{I} \sim q^{\dagger} \vec{\Gamma} \cdot \vec{\pi} q$, where $\vec{\Gamma}$ is a matrix that contains the isospin and spin information. The effective coupling at each vertex is proportional to $\left\langle H_{I}\right\rangle / \sqrt{N_{c}}$, where $\langle\cdots\rangle$ represents the nucleon matrix element and $1 / \sqrt{N_{c}}$ is the normalization factor needed for each external pion. Since a color-singlet nucleon contains $N_{c}$ quarks, $\left\langle H_{I}\right\rangle \sim N_{c}$, so the effective coupling at each vertex is of order $\sqrt{N_{c}}$, making each tree amplitude $\sim N_{c}$. However, the term proportional to $N_{c}$ is canceled in the sum of the two diagrams, leaving a total amplitude $\left\langle U_{2}\right\rangle$ of order unity in the large- $N_{c}$ limit. This can be understood [9] directly from (3) firstly because $\left\langle K_{1}\right\rangle=0$ on account of energy-momentum conservation: an on-shell nucleon 
cannot absorb or emit a massive pion and remains on-shell. Thus $\left\langle U_{2}\right\rangle=\frac{1}{2}\left\langle K_{2}\right\rangle$. Since the commutator of two one-body operators is again a one-body operator, hence $\left\langle\left[H_{1}, H_{2}\right]\right\rangle \sim$ $\left\langle q^{\dagger}\left[\vec{\Gamma}_{1} \cdot \vec{\pi}_{1}, \vec{\Gamma}_{2} \cdot \vec{\pi}_{2}\right] q\right\rangle \sim N_{c}$. Taking into account the normalization factor $1 /{\sqrt{N_{c}}}^{2}$ for two pions, we conclude that $\left\langle U_{2}\right\rangle=\frac{1}{2}\left\langle K_{2}\right\rangle \sim N_{c} / N_{c}=1$, as needed. Like the situation in Example 1, again there is no need to cancel the $N_{c}$ term explicitly, because the commutator structure of $K_{2}$ already provides for it. Physically, the one-body nature of $K_{2}$ tells us that the two pions must interact with the same quark in the nucleon.

With these two examples, it is clear that the commutator structure of $K_{2}$ is the key to the simplification. The question is whether $K_{n}$ still possesses such commutator structures for higher $n$. The answer is 'yes', though the detailed structure is increasingly more complicated. Let us define anti-hermitean operators $C_{n}$ to have the simple nested commutator structure,

$$
C_{n}=(-i)^{n} \int_{\mathcal{R}_{n}} d^{n} t\left[H_{1},\left[H_{2},\left[\cdots,\left[H_{n-1}, H_{n}\right] \cdots\right]\right]\right], \quad \text { cn }
$$

where $H_{i} \equiv H_{I}\left(t_{i}\right)$. Then it can be shown [10] that

$$
U_{n}=\sum_{(m)} \prod_{j=1}^{k} \frac{1}{\sum_{i=j}^{k} m_{i}} C_{m_{1}} C_{m_{2}} \cdots C_{m_{k}}, \quad \text { xic }
$$

where the sum is taken over all partitions $(m)=\left(m_{1} m_{2} \cdots m_{k}\right)$ of the number $n$. In particular, $U_{1}=C_{1}=K_{1}, U_{2}=\left(C_{1}^{2}+C_{2}\right) / 2=\left(K_{1}^{2}+K_{2}\right) / 2, U_{3}=\left(C_{1}^{3}+C_{1} C_{2}\right) / 6+$ $\left(C_{2} C_{1}+C_{3}\right) / 3=K_{1}^{3} / 6+\left(K_{1} K_{2}+K_{2} K_{1}\right) / 4+K_{3} / 3$. From these relation, or more generally by comparing (11) with (5), one obtains the commutator structure of $K_{n}$ to be $K_{n}=C_{n}+R_{n}$, with $R_{1}=R_{2}=0, R_{3}=\left[C_{2}, C_{1}\right] / 4$, and $R_{4}=\left[C_{3}, C_{1}\right] / 3$. In general, $R_{n}$ is given by commutators of $C_{m}$, with increasingly complicated coefficients as $n$ increases.

We can use these relations between $U_{n}$ and $C_{m}$ to calculate quark-quark and electronelectron scatterings to higher orders, avoiding delicate cancelations, and obtaining directly the energy dependence necessary for reggeon structures in QCD, viz., an amplitude proportional to $g^{2 k}\left(g^{2} \ln s\right)^{n-k} G_{k}$ when $k$ color-octet objects are being exchange in the $t$-channel [6.11]. They can also be used to obtain the correct amplitude [12] $\sim N_{c}^{1-n / 2}$ for $p i$-nucleon inelastic scattering with $n-1$ pions in the final state, without having to deal with delicate 
cancelation of $n-1$ powers of $N_{c}$ encountered by summing Feynman tree diagrams [9]. As mentioned at the beginnig, abelian and non-abelian eikonal approximations are special cases of these formulas. Infrared structure of QED can be obtained from these formulas, with $K_{1}$ containing the Bloch-Nordsieck result, $K_{2}$ giving rise to the Coulomb phase, and $K_{n}=0$ for $n \geq 3$ [13,14]. The Landau-Pomeranchuk-Migdal effect [15] can also be cast into this form 16] with $K_{n}=0$ for $n \geq 3$. Other applications may require knowing the the diagrammatic rules for calculating $K_{n}$. For QED such rules are known [14. For tree diagrams in QCD involving an energetic particle such rules are also known [6], but the general case for QCD is still to be worked out.

So far we have emphasized situations when $U$ is the time-evolution operator with a known Hamiltonian. If the dynamics is unknown, we can still use $K_{n}$ in (1), or $C_{n}$ in (5), to parametrize the unitary dynamics. As long as $K_{n}$ is anti-hermitean, it is clear that $U$ is unitary. As long as $C_{n}$ is anti-hermitean, it can also be shown [17 that $U$ is unitary, though the proof is much more involved. A Wolfenstein-like parametrization of the CKM matrix to all orders can be deduced from these parametrizations. They can also be used to study the unitary matrix describing the overlap of the unperturbed and the perturb energy eigenfunctions [17].

In conclusion, we have given a number of examples to show that the unitary perturbation theory, in which $K=\ln U$ is expanded perturbatively, is in many ways better than the ordinary perturbation theory, where $U$ is directly expanded.

This research is supported in part by the Natural Sciences and Engineering Research Council of Canada, and the Fonds pour la formation de Chercheurs et l'Aide à la Recherche of Québec. I am indebted to Saad Ali, Hong-Mo Chan, Marc Grisaru, Greg Mahlon, SheungTsun Tsou, Wu-ki Tung, and Kenneth Young for stimulating discussions. 


\section{REFERENCES}

[1] W. Magnus, Comm. Pure Appl. Math 7 (1954) 647.

[2] See, for example, Kerson Huang, 'Statistical Mechanics' (John Wiley and Sons, 1963).

[3] See, for example, J.J. Sakurai, 'Modern Quantum Mechanics', (Addison-Wesley, 1994).

[4] P. Kulish and L.D. Faddeev, Teor. Pat. Fiz. 4 (1970) 153 [Theor. Math. Phys. 4 (1971) 745.]

[5] R. Torgerson, Phys. Rev. 143 (1966) 1194; H. Cheng and T.T. Wu, Phys. Rev. 182 (1969) 1868, 1899; M. Levy and J. Sucher, Phys. Rev. 186 (1969) 1656.

[6] Y.J. Feng, O. Hamidi-Ravari, and C.S. Lam, Phys. Rev. D 54 (1996) 3114.

[7] S. Ali, M.Sc. thesis, McGill University (2000).

[8] H. Cheng and T.T. Wu, 'Expanding Protons: Scattering at High Energies', (M.I.T. Press, 1987).

[9] C.S. Lam and K.F. Liu, Phys. Rev. Lett. 79 (1997) 597.

[10] C.S. Lam, J. Math. Phys. 39 (1998) 5543.

[11] Y.J. Feng and C.S. Lam, Phys. Rev. D 55 (1997) 4016; R. Dib, J. Khoury, and C.S. Lam, Phys. Rev. D 60 (1999) 036001.

[12] G. 't Hooft, Nucl. Phys. B72 (1974) 461; E. Witten, Nucl. Phys. B160 (1979) 57;

S. Coleman, Erice Lectures (1979), in 'Aspects of Symmetry' ( Cambridge University Press, 1985).

[13] F. Bloch and A. Nordsieck, Phys. Rev. 52 (1937) 54; M. Ciafaloni, Phys. Lett. 150B (1985) 379; S. Catani and M. Ciafaloni, Nucl. Phys. B249 (1985) 301, B264 (1986) 588; C.S. Lam, J. Math. Phys. 41 (2000) 4497.

[14] H.D. Dahmen, W. Krzyzanowski, and M.L. Larsen, Phys. Rev. D 33 (1986) 1726. 
[15] L.D. Landau and I.J. Pomeranchuk, Dokl. Akad. Nauk. SSSR 92 (1953) 535, 92 (1953) 735; A.B. Migdal, Phys. Rev. 103 (1956) 1811; R. Blankenbecler and S.D. Drell, Phys. Rev. D 53 (1996) 6265; R. Baier, Yu.L. Dokshitzer, A.H. Mueller, S. Peigné, and D. Schiff, Nucl. Phys. B483 (1997) 291.

[16] I.M. Dremin and C.S. Lam, Mod. Phys. Lett. A 13 (1998) 2789.

[17] C.S. Lam, to be published. 


\section{FIGURES}

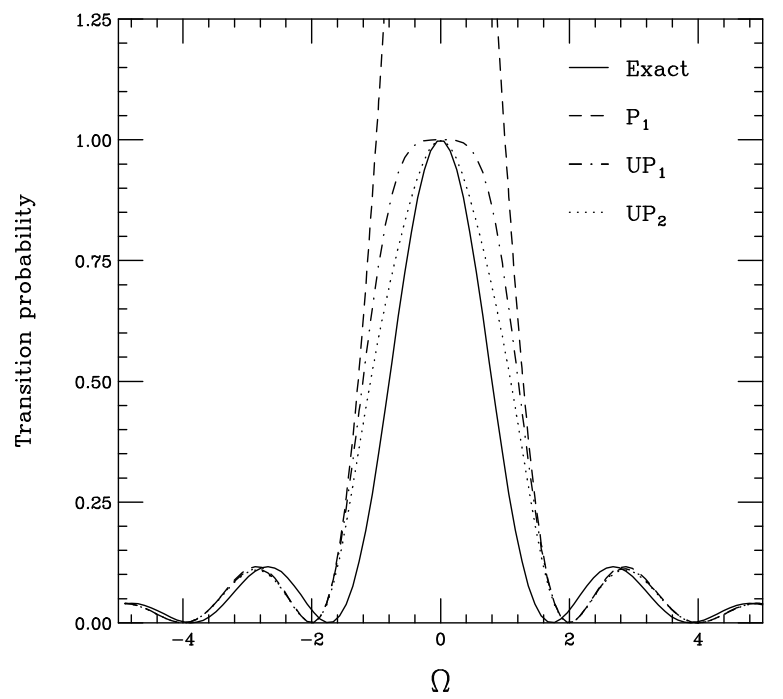

FIG. 1. Unitary perturbation theory vs ordinary perturbation theory in a two-level example.

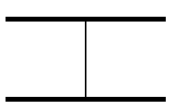

(a)

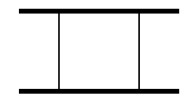

(b)

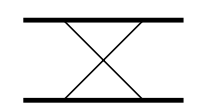

(c)

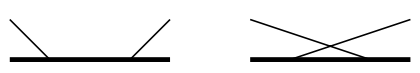

(d)

(e)

FIG. 2. (a,b,c), high-energy quark-quark scattering diagrams to one loop order. (d,e), meson-nucleon scattering at large $N_{c}$ in the tree approximation . 